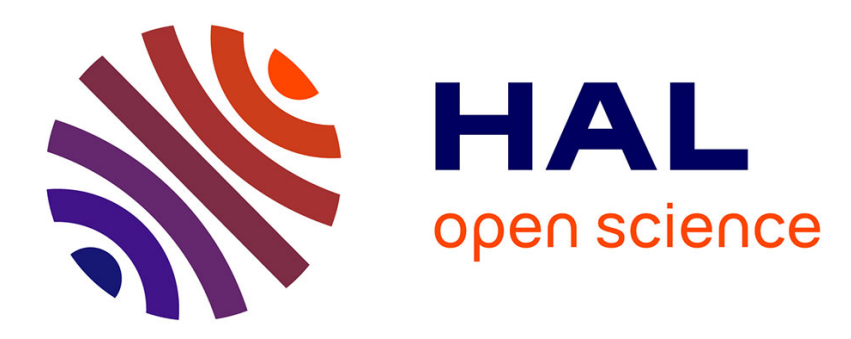

\title{
La transition néolithique : la contribution de la génétique
}

Jacques Chiaroni, Aurore Schmitt

\section{To cite this version:}

Jacques Chiaroni, Aurore Schmitt. La transition néolithique : la contribution de la génétique. CORPS : Revue Interdisciplinaire, 2019, №17 (1), pp.143. 10.3917/corp1.017.0143 . hal-02991002

\section{HAL Id: hal-02991002 \\ https://hal.science/hal-02991002}

Submitted on 5 Nov 2020

HAL is a multi-disciplinary open access archive for the deposit and dissemination of scientific research documents, whether they are published or not. The documents may come from teaching and research institutions in France or abroad, or from public or private research centers.
L'archive ouverte pluridisciplinaire HAL, est destinée au dépôt et à la diffusion de documents scientifiques de niveau recherche, publiés ou non, émanant des établissements d'enseignement et de recherche français ou étrangers, des laboratoires publics ou privés. 


\title{
La transition néolithique : la contribution de la génétique
}

\author{
Jacques Chiaroni et Aurore Schmitt
}

Le néolithique est une période charnière de l'histoire et de l'évolution bio-culturelle de l'Homme. Bien que la signification du terme Néolithisation évolue au cours du temps et reflète l'évolution des concepts de la discipline archéologique, nous retiendrons qu'il s'agit d'une transformation progressive du mode de subsistance de nombreux groupes humains qui passent d'un mode de chasseurs-cueilleurs nomades (économie de prédation) à un mode d'agriculteurs-éleveurs sédentaires (économie de production). Considérée de nos jours comme une période de «transition » plutôt que de «révolution », le processus de néolithisation s'est déroulé sur plusieurs millénaires sur plusieurs régions du globe de façon indépendante (Afrique saharienne, Mésoamérique, Amérique du sud, Nouvelle Guinée, Chine). C'est dans le croissant fertile, au Proche Orient, que cette transition est survenue le plus tôt, aux alentours de 9500 ans avant J.-C. pour diffuser ensuite en Méditerranée et en Europe continentale. Face à la complexité d'un tel phénomène, pour tenter de le comprendre, de nombreuses disciplines sont mises à contribution. C'est le cas de la génétique qui étudie notamment les modalités de peuplement et permet d'apporter des éléments au débat sur la diffusion démique ou culturelle du Néolithique du Proche-Orient à l'Europe occidentale.

\section{LES GRANDES ETAPES DE LA TRANSITION NEOLITHIQUe AU PROCHE ORIENT (Demoule 2010)}

Le croissant fertile s'étend entre le Levant méditerranéen à l'ouest, le plateau anatolien au nord et les montagnes du Zagros à l'est englobant la plaine mésopotamienne délimitée par les fleuves Tigre et Euphrate s'unissant au sud pour terminer dans le Golfe Persique (figure 1). $\mathrm{Au}$ sud de ce croissant, le désert. C'est dans cette région du Proche Orient que, vers 9500 avant J.-C., apparaissent les premiers signes de la néolithisation.

Figure 1 : le croissant fertile (DAO : J. Chiaroni).

Les derniers chasseurs-cueilleurs nomades appartiennent à la culture archéologique de Kebara (17 000-12 000 av. J.-C.) localisée autour du lac de Tibériade et caractérisée par une production de microlithes géométriques. C'est entre 12500 et 10000 av. J.-C. que l'on retrouve les premières traces de sédentarisation en petits hameaux sur des sites attribués à la culture Natoufienne. Les Natoufiens sont des chasseurs-cueilleurs subsédentarisés autour du Jourdain et de l'Euphrate mais certains de leurs outils indiquent une exploitation des ressources végétales (faucille, meules...). La question du mode de subsistance des Natoufiens est liée à celle de l'émergence de l'agriculture mais est loin d'être résolue.

Par la suite, le PPNA (Pre Pottery Neolithic A ; 9700 - 8500 av.J.-C.) et PPNB (8500 - 6700 av.J.-C.) sont des périodes fastes qui se caractérisent par la présence de sites de grande taille localisés dans les régions les plus fertiles (la vallée du Jourdain, par exemple). Ces sites occupés tout au long de l'année permettent le développement de la domestication des plantes (PPNA) puis des animaux (PPNB). On voit également apparaître au PPNB les premières structures communautaires de grande taille (Mithen 2006).

Cette période est marquée par la construction de temples monumentaux comme à Jericho ou Göbekli Tepe. Ce dernier, situé au sud du Taurus oriental entre Euphrate et Tigre, est caractérisé par des stèles imposantes de plus d'une dizaine de tonnes sculptées de représentations d'animaux souvent dangereux (taureaux, fauves, scorpions) pouvant évoquer la guerre (Testart 2010). Ces populations conservent toujours une forte activité de chasse et de cueillette, une spécialisation en fonction de l'environnement avec de l'agriculture et de 
l'élevage dans les régions humides, de l'élevage avec parfois un pastoralisme nomade dans les régions arides ou semi arides (figure 2). Mais on note toujours une absence de céramique alors qu'ils sont des agriculteurs accomplis.

Figure 2: spécialisation des activités agriculteurs dans les zones humides, éleveurs nomades dans le zones arides (DAO : J. Chiaroni).

Ce n'est qu'à la fin du $8^{\text {ième }}$ millénaire qu'apparait la céramique d'abord dans la vallée d'Amuq au nord d'Antioche puis en Mésopotamie. On note donc que, si en dehors du Proche Orient, la céramique est un paramètre qui définit la Néolithisation, ce n'est pas le cas ici où son apparaition est même plus tardive que dans d'autres endroits du monde comme au Japon par exemple (17 000-15 000 av.J.-C.). ainsi, au Proche Orient, l'apparition de la céramique n'est pas liée à la Néolithisation.

\section{LES HYPOTHESES EXPLIQUANT LA NEOLITHISATION ${ }^{i}$}

Si les lieux et dates de transition sont de mieux en mieux documentés, les fondements d'un tel changement sont toujours discutés. L'apparition d'un environnement plus propice lié au rechauffement faisant suite à la dernière glaciation a été considérée comme le facteur déclenchant d'un changement de mode de vie. Mais le phénomène est plus complexe.

Dès 1925, Childe introduit l'expression "révolution néolithique" en analogie à la révolution industrielle. De cette manière, il inscrit cette étape de la préhistoire dans une conception économique de l'évolution des sociétés. Le système économique fondé sur la chasse et la cueillette est abandonné pour l'agriculture et l'élevage. La domestication des plantes et des animaux impliquait l'adoption d'un mode de vie sédentaire, un essor démographique et l'apparition d'inégalités sociales. Malgré le fait que cette hypothèse soit largement contredite, elle appartient à l'histoire de l'Archéologie.

Jacques Cauvin (1994), quant à lui, assimile la transition néolithique à une véritable révolution symbolique. L'image mentale horizontale du monde des chasseurs-cueilleurs, où l'homme est intégré au même niveau que les autres êtres vivants, aurait évolué vers une verticalisation de cette image avec un positionnement de l'homme entre le divin et le reste de la nature. Les chasseurs-cueilleurs possèdaient l'aptitude à domestiquer mais dans un souci d'échange avec la nature et non pas de domination. Les populations néolithiques seraient finalement des intermédiaires entre le «tout sauvage» du Mésolithique et le «tout domestique » de l'âge du fer. Ainsi, ce serait cette position intermédiaire de l'homme qui aurait poussé celui-ci à dominer la nature et à évoluer vers une économie de production.

Alain Testart (2012) propose une autre analyse. Ce n'est pas l'agriculture mais la sédentarité permettant le stockage qui aurait été l'élément fondamental de la Néolithisation. Productionde céramique et sédentarité semble précéder l'agriculture. Les Natoufiens auraient été des chasseurs-cueilleurs, sédentaires, stokeurs. La sédentarité se présente comme la condition préalable à l'accumulation de biens matériels, au développement d'équipements lourds, mais aussi à la construction de diverses structures bâties. Elle rend possible un développement de la richesse. Par la conservation rendue possible, le stockage transforme la nourriture en bien durable accumulable. Convertir en biens matériels un excédent de nourriture permet d'accéder à une « richesse » qui donne lieu à une différenciation entre ceux qui la possèdent et ceux qui ne la possèdent pas. Ce processus implique des inégalités au sein d'un groupe. La production de l'inégalité serait donc le moteur de la Néolithisation. 


\section{LA DIFFUSION DU « PACKAGE « NEOLITHIQUE VERS LA MEDITERRANEE ET L'EUROPE}

La mise en place de l'économie de production a pris plusieurs millénaires. Celle-ci se caractérise par la sédentarité (fixation dans l'espace en un lieu unique), le développement de l'agriculture et la domestication des animaux avec le développement de l'élevage ${ }^{\mathrm{ii}}$, et l'invention de la céramique qui constitue le matériau de prédilection pour la fabrication de récipients (stockage, préparation et consommation de la nourriture). La nécessité d'aménager des champs pour pratiquer l'agriculture conduit à fabriquer des haches pour abattre des arbres et donc au polissage de la pierre. Ces cinq éléments constituent le package néolithique ${ }^{\text {iii }}$.

$\mathrm{Au} 7^{\text {ième }}$ millénairre avant notre ère, la panoplie néolithique est acquise et se diffuse en dehors $\mathrm{du}$ foyer Proche Oriental (Guilaine 1996). Les bases de cette expansion sont toujours discutées afin d'en définir les raisons (pressions démographiques, épuisements des sols, changement climatique, pression culturelle ....), les modalités (une diffusion des idées (acculturation) ou d'une diffusion des hommes (expansion démique)) et les effets lors de la rencontre avec les populations locales s'il s'agit d'une expansion démique (absorption, remplacement ...). L'une des premières expansions touche l'ensemble de la Turquie entre 8000 et 7000 av.J.-C et la péninsule balkanique qui est atteinte vers 6500 av.J.-C.

A partir de là, deux courants de diffusion vers l'Europe ont été identifiés (figure 3). Le courant méditerranéen qui longe les côtes et atteint d'abord la Sicile, la Sardaigne, la Corse et les côtes méditerranéennes de l'Europe occidentale puis la façade atlantique de la péninsule ibérique aux alentours de 5400 av.J.-C.. Il est a noter qu'à Majorques et aux Baléares les témoignages néolithiques sont attestés plus tard, vers 4000 av.J.-C. Ce courant est caractérisée par une poterie décorée par impression de poinçon ou de coquillage, le Cardium edule d'où le nom qui lui est attribué «Céramique impresso-cardial ». Le second courant se dirige vers l'Europe tempérée centrale puis occidentale à partir de 5500 av.J.-C. Les dernières expansions se situent dans les iles britanniques et la Scandinavie vers 4000 av.J.-C.. Cette expansion définie une aire culturelle caractérisée par une céramique rubanée (Linearbandkeramic LBK) dans les vallées du Danube, de l'Elbe et du Rhin.

Figure 3 : les expansions néolithiques en Europe de l'Ouest (DAO : J. Chiaroni).

D'un point de vue culturel, si cette diffusion s'est déroulée sur plusieurs siècles, le package néolithique a fini par l'emporter sur les modes de subsistance des populations locales.

\section{QUELS SONT LES IMPACTS BIO-CULTURELS DE CETTE TRANSITION NEOLITHIQUE ?}

La Néolithisation a eu des effets tant biologiques que culturels sur les populations anciennes. A partir du Néolithique, suite à la diminution de la mobilité, une tendance générale à la gracilisation du squelette est observée (Ruff et al. 2015). L'augmentation de la démographie dont on ne sait si elle est un facteur déclenchant ou une conséquence de la Néolithisation s'oberve sur l'ensemble de la planète (Crubézy et al. 2008). A côté de l'alimentation d'autres facteurs comme la sédentarisation, une puberté plus précoce et une disponibilité du lait animal ont pu avoir des actions synergiques. Toutefois, il a été démontré que des succès démographiques ont pu survenir indépendemment de l'agriculture comme c'est le cas pour l'expansion démographique des ancêtres des agriculteurs de langue bantoue qui date de 10000 ans et qui semble avoir précédé la transition néolithique dans cette partie de l'Afrique (Quintana-Murci \& Patin 2018).

La Néolithisation a également eu un effet sur la santé des populations (Larsen 2010). Paradoxalement ces changements ont généré un certain nombre d'effets négatifs: alimentation moins diversifiée et risque de carence, pathologies infectieuses favorisées par une démographie sédentaire plus importante (rougeole, variole) ainsi que par des contacts plus 
étroits avec les animaux domestiqués ou des rongeurs attirés par les stocks de nourriture (zoonoses) et enfin une accumulation des eaux usées (choléra). Le paludisme, dans certaines zones climatiques, a probablement vu un essor lié aux pratiques agricoles de déforestation et stagnation d'eau dans des canaux d'irrigation ainsi qu'à la sédentarisation d'un effectif humain plus important facilitant «le travail » des vecteurs.

Ce changement de mode de subsistance a également eu des conséquences sur l'organisation des groupes humains qui ont donné lieu à de multiples théories, telles qu'une aumentation de la hiérarchisation sociale et des violences inter-personnelles. Actuellement, les impacts sociaux de la Néolithisation sont considérés comme des phénomènes complexes qui nécessitent d'englober de nombreux paramètres (Testart 2012; Jeunesse 2018). Les interprétations varient entre disciplines et traditions académiques.

Quoi qu'il en soit, les changements liés à la Néolithisation sur l'Homme d'un point de vue bio-culturelle nécessite des études multidisciplinaires. La génétique a donc son rôle à jouer pour comprendre, par exemple, les effets d'un changement de régime alimentaire ou les modalités du mode de diffusion de ce nouveau mode subsistance.

\section{QUELQUES CONTRIBUTIONS DE LA GENETIQUE}

Cas particulier de la persistance de la lactase (Quintana-Murci et Patin, 2018)

Il s'agit d'un exemple d'adaptation de l'homme à son régime alimentaire. La lactase est une enzyme qui permet de digérer le lactose du lait. Cette fonction est particulièrement importante au cours des premiers mois de vie. Par la suite, lors de l'introduction d'une alimentation diversifiée, de façon physiologique son activité diminue. Ainsi les individus porteurs de cette forme ancestrale ne sont capables de digérer le lait que durant cette période néonatale. Par la suite des mutations de ce gène associées à la persistance de cette lactase à l'age adulte a vu sa fréquence augmenter de façon significative dans les populations où l'élevage est (ou a été) une ressource nutritionnelle importante. Cela pourrait être considéré comme un exemple d'interaction entre culture (élevage) et biologie (sélection de la mutation génétique) où la première crée de nouveaux environnements qui conduisent à de nouvelles pressions de sélection sur les gènes. D'une façon générale, les changements de mode de subsistance ont permis aux gènes d'être épargnés par la sélection ou d'être soumis à de nouvelles sélections. De même, la pluralité des mutations décrites spécifiques de certaines populations peut être considérée comme un exemple de convergence évolutive permettant d'accéder à un même résultat bénéfique par divers chemins.

\section{La contribution des marqueurs haploïdiques : le chromosome Y et l'ADN mitochondrial}

La variabilité génétique au sein des populations actuelles représente de véritables archives du passé. Chez l'homme, cette information est conservée dans son génome qui possèdent 3 milliards de bases dont seulement $0.33 \%$ (10 millions) permettent de différencier les individus entre eux et seulement 3 millions constituent nos 25000 gènes. Ce génome est composé de 46 paires de chromosomes localisés dans le noyau (22 paires d'autosomes, 1 paire de chromosomes sexuels avec XY chez l'homme et XX chez la femme) et de l'ADN mitochondrial.

Dans ce chapitre nous nous concentrerons sur un type de polymorphisme particulier dénommé SNV (single nucleotide variation ${ }^{\text {iv }}$ ) qui est caractérisé par la substitution d'un seul nucléotide ne survenant que très rarement, à priori une seule fois, au cours de l'histoire humaine. La particularité de ces marqueurs en font un outil de choix à deux niveaux. Le premier niveaux concerne la génétique des populations à proprement parlé. En effet la mesure de la fréquence d'un grand nombre de SNV autosomaux permet d'estimer le degré d'affinité entre des populations. Il existe des algorithmes, traitant de grandes quantités de données SNV, qui permettent de révéler des signaux de structuration et de mélange de populations. Toutefois, ils 
ne peuvent pas estimer la date de ces événements et la directionnalité d'un flux génétique. Or la connaissance de la date et de la durée d'un évènement sont des informations majeures pour un archéologue. Ainsi, le deuxième niveaux d'intérêt de ces marqueurs concerne la phylogénie. Il est en effet possible de rattacher des SNV localisés sur un même chromosome (et qui n'ont pas subit de phénomènes de recombinaisons) à un ancêtre commun. La reconstruction phylogénétique ne peut donc pas se faire à partir des autosomes et des chromosomes $\mathrm{X}$ où le processus de recombinaison fractionne les relations originelles entre les SNV d'un même chromosome. Il est important que ces mutations demeurent alignées ensemble comme des perles sur un collier qui seront alors transmises en bloc du passé au présent. Ce maintien groupé des mutations qui s'accumulent de génération en génération permet alors une construction hiérarchique ou phylogénétique de ces différents marqueurs, du plus ancestral au plus dérivé. Il convient donc de sélectionner des régions génomiques qui échappent au processus de recombinaison comme le chromosome Y et l'ADN mitochondrial qui, en maintenant l'accumulation séquentielle de ces SNV sur un même chromosome, permettent la construction d'un arbre phylogénique relativement robuste et dont les nœuds peuvent être datées.

Cette datation repose sur l'hypothèse d'horloge moléculaire qui suppose que l'évolution de la séquence d'ADN, et notamment l'apparition de SNV, se produit à un rythme constant. Cette hypothèse assume donc une linéarité entre le pourcentage de différences (mutations) et le temps de divergence depuis l'ancêtre commun. On parle du temps de coalescence. On conçoit que les haplotypes autosomaux soumis au processus de recombinaisons voient cette information se dégrader en quelques milliers d'années. Les systèmes haploïdes (ADN mitochondrial et chromosome Y) sont donc à ce titre des candidats privilégiés.

L'ADN mitochondrial (ADNmt) est un ADN circulaire de 16500 paires de bases présent dans chaque mitochondrie et qui comporte un certain nombre de gènes impliqués dans les processus énergétiques de la cellule. La transmission se faisant de mère à enfants, son étude reflète ainsi l'histoire des femmes. C'est sa localisation dans la pièce intermédiaire du spermatozoïde qui restant, après fécondation, à l'extérieur de l'ovule explique que les hommes ne contribuent pas à cet héritage. L'ADN mitochondrial présente un certain nombre d'avantages : les deux sexes ont des génomes d'ADNmt (plus d'échantillons incluant de l'ADN ancien), il existe un nombre élevé de molécules d'ADNmt par cellule (contrairement au chromosome $\mathrm{Y}$ qui est en copie unique), il existe de nombreuses données qui sont répertoriées dans des banques de données accessibles à tous. Les inconvénients de ce génome sont liés à sa petite taille, au taux élévé de mutations donc au risque de récurrence ajoutant de l'incerttitude et enfin à l'existence de gènes sensibles à la sélection naturelle pouvant aboutir à une réduction de la diversité en dehors de tout évènment démographique.

Le chromosome $\mathrm{Y}$ comporte 50 millions de paires de bases avec peu de gènes. La transmission se faisant de père à fils, son étude reflète ainsi l'histoire des hommes. Sa diversité ne semble être façonnée que par des forces démographiques, croissance populationnelle, effet fondateur ou migrations. Un autre avantage du chromosome Y est lié à la taille de son génome puisqu'aujourd'hui, avec l'arrivée du séquençage de nouvelle génération, les relations phylogénétiques entre les différentes lignées (haplogroupe) du Y sont basées sur 1100 SNV alors que celles de l'ADMmt ne sont établies qu'à partir de 50 SNV, soit 20 fois moins (Roy J. King \& Peter A. Underhill. 2018). Il a donc été possible de créer un phylogénie calibrée (Figure 4) en fonction du temps dont la fiabilité a été renforcée par l'apport de l'ADN ancien. Sur la base d'un séquençage de 10 millions de bases il a été possible d'évaluer le taux moyen auquel les SNV sont ajoutés au hasard au cours de l'évolution. On estime ce taux à $1 \mathrm{SNV}$ tous les 132 ans (Poznik, G. D, 2016). Il est donc possible de définir le temps de coalescence (TMRCA : Time to the Most Recent Common Ancestor) qui est le temps qu'il faut pour remonter dans le temps et arriver à l'ancêtre 
commun. La longueur des branches étant proportionnelle au temps écoulé, si une branche est très longue il est possible que d'autres branches aient été perdues et s'il existe de nombreuse branches rayonnant à partir d'une branche ancestrale il est possible que cela soit le fait du début d'une expansion démographique rapide à partir d'un goulot d'étranglement antérieur (Roy J. King \& Peter A. Underhill. 2018). Ainsi, le phylogénéticien décortique les différentes hypothèses de relations entre lignées (branches de l'arbre) et c'est l'ajout de certains individus (ancien ou actuel) dans la phylogénie qui va permettre parfois de confirmer certains scénarios évolutifs. On peut citer l'exemple issu de l'ajout de l'ADN d'un spécimen sibérien d'environ 45000 ans (Ust'-Ishim) qui a permi de confirmer l'hypothèse de perte de branches (extinctions de lignées) (Fu, Q, 2014) ou encore l'exemple de l'existence de relations entre lignées comme illustrées par l'haplogroupe A00 (Mendez F.L, 2016) qui est le témoin d'un phénomène d'introgression d'ADN ${ }^{v}$ qui s'est produit entre un mâle hominoïde archaïque et une femme anatomiquement moderne.

\section{Figure 4 d'après King $\&$ Underhill $^{8}$}

Comme nous l'avons vu, si ces deux types de marqueurs permettent la construction d'une phylogénie relativement robuste, ils ne sont le reflet que des récits féminins ou masculins dans l'histoire des migrations humaines et peuvent être aussi sujets à exagération ${ }^{8}$ dans les ampleurs de certains évènements démographiques en raison notamment de leur sensibilité au phénomène de dérive génétique (la diversité perdue ou maintenue au fil des générations par le seul fait du hasard). En effet la constitution du patrimoine génétique d'un enfant à partir d'un couple de parents se fait avec 4 autosomes recombinés (mosaïque ancestrale), 3 chromosomes $\mathrm{X}$ recombinés, 1 copie de chromosome $\mathrm{Y}$ et une copie d'ADN mitochondrial tous deux non recombinés. Ainsi, dans des conditions de neutralité (c'est-à-dire sans effet de sélection), une nouvelle mutation du chromosome $\mathrm{Y}$ apparaissant pour la première fois dans le pool génétique a au moins quatre fois plus de chance de s'établir, par hasard seulement, dans une population que toute nouvelle mutation qui survient sur l'un des 4 autosomes.

Il en est de même des limites de l'extrapolation d'événements préhistoriques fondés uniquement sur des données d'ADN modernes pour lesquelles des épisodes démographiques antérieurs ont pu être remplacés par des événements plus récents dont le fait le plus marquant est représenté par l'extinction des signaux génétiques liés à l'expansion de l'agriculture en Europe (Haplogroupe G) par des vagues migratoires plus récentes survenues au cours des derniers 5000 ans en provenance des steppes (haplogroupe R1b) (voir ci-après) Dans ces cas les données de l'ADN ancien (incluant les espèces archaïques) et l'approche multidisciplinaire de ces études sont d'un apport fondamental.

Génomique des populations actuelles et ADN ancien ont donc fortement enrichi le domaine de l'archéogénétique. Les nouvelles capacités de séquençage ont notamment affiné les données concernant le chromosome $\mathrm{Y}$ en particulier le calibrage temporel de son arbre phylogénique. Ces nouveaux éléments permettent de mieux explorer des hypothèses spécifiques de l'histoire de l'Homme et notamment celles en lien avec la transition néolithique pour laquelle nous allons abordé quelques questions vues par le chromosome $\mathrm{Y}$.

\section{Que sont devenues les populations indigènes d'Europe d'avant la Néolithisation ?}

Il semble que ces populations aient été remplacées. En effet, deux squelettes mâles du paléolitiques, un spécimen russe d'environ 37000 ans (Kostenki 14) et un spécimen belge de 35000 ans (Goyet 116.1), possédaient une variété apparentée de l'haplogroupe C du chromosome Y (Seguin-Orlando, A., 2014 et Fu, Q., 2016). Ce même haplogroupe a également été détecté chez un Ibérique du mésolithique de 7000 ans (La Braña 1) (Olalde, 
2014). Or, cette variété particulière d'haplogroupe $C$ est presque absente des populations actuelles de l'Europe (figure 5) (Chiaroni J, 2009).

Figure 5 : répartition de l'haplogroupe $\mathrm{C}$ du chromosome $\mathrm{Y}$

La Néolithisation de l'Europe: une diffusion démique ou culturelle (ou les deux) ?

Nous l'avons vu, cette question qui fait toujours débat. L'archéogénomique dans ce domaine peut être un outil déterminant en détectant la présence de signaux génétiques en provenance du Proche Orient à partir d'ADN extrait de restes humains dont la chronologie est archéologiquement maitrisée. Cette présence, surtout si elle est identifiée sur plusieurs squelettes, permettra de confirmer une expansion démique sans pour autant éliminer un phénomène d'acculturation associé. En fonction des aires géographiques considérées des points peuvent être spécifiquement discutés.

- Au Proche Orient. La transition néolithique semble être liée à des populations génétiquement diverses qui habitaient la région. En effet, le séquençage d'une femme du Néolithique précoce du site de Ganj Dareh (Gallego-Llorente M, 2016), dans les montagnes de Zagros en Iran présentant les indices d'une économie basée sur l'élevage caprin remontant à 10.000 ans avant notre ère, a démontré la présence de deux lignées distinctes; les agriculteurs anatoliens et les éleveurs de montagne de Zagros. Ce constat soulèvant d'ailleurs la question quant à l'indépendance des innovations survenant dans des lieux différents.

- En Mer Egée. Il apparait que les populations néolithique du nord de la mer Égée peuvent avoir été le produit des migrations en provenance d'Anatolie et du Levant et de groupes locaux mésolitiques égéens ayant adopté l'agriculture. Ces scénarios sont d'ailleurs cohérents avec la diversité des cultures matérielles des communautés néolithiques égéennes et l'héritage d'un savoir-faire local notament en termes de fourrage (Kılınç GM, 2017).

- En méditerranée occidentale (Roy J. King \& Peter A. Underhill. 2018). Des données concernant l'ADN actuel et ancien du chromosome Y démontrent que Corses et Sardes contemporains conservent le patrimoine génétique néolithique qui reflète le mieux l'expansion démographique des agriculteurs en Europe (Keller, A., 2012). En effet, la momie Otzi («iceman») porte l'haplogroupe G2a-L91, une branche de l'haplogroupe précédent G2a-PF3147. Ces deux haplogroupes, étroitement apparentés, montrent leurs fréquences les plus élevées en Sardaigne et dans le sud de la Corse alors qu'ils ne représentent qu'une faible fréquence par ailleurs $(\sim 1 \%)$ y compris en Anatolie et à Chypre (Voskarides, K., 2016). Or la présence de lignées apparentées au PF3147 dans l'ADN ancien de spécimens néolithiques d'Anatolie occidentale (Mathieson I., 2015) ainsi que dans des spécimens néolithiques du Sud de la France (Lacan, M., 2011) est compatible avec un flux génétique associé dans la région méditerranéenne à la transition néolithique. De plus, une analyse autosomique a également montré que l'affinité d'Iceman était plus proche des Sardes modernes que des autres Européens continentaux modernes (Keller, A., 2012). Enfin il convient de signaler que ces lignées apparentées au PF3147 sont totalement absentes dans les spécimens d'ADN anciens de l'ère mésolithique postglaciaire (Hofmanová, Z., 2016). Les données de l'ADN ancien, malgré l'absence de clines de fréquence dans les populations actuelles, soutiennent donc l'hypothèse d'une Néolithisation par expansion démique (figure 6).

Figure 6 : fréquence de l'haplogroupe G-L91 (Keller, A., 2012) 


\section{Que sont devenu les populations du Néolithique en Europe?}

Leur faible fréquence actuelle indique que ces lignées G2a spécifiques ont été remplacées par des populations de steppes eurasiennes en expansion rapide associées à l'âge du bronze (Haak, W. 2015), dont la composition se rapproche de la structure génétique des populations européennes observée aujourd'hui (R1b et R1a pour le chromosome Y), avec certains éléments reconnus dans les cultures de l'âge du fer (Gamba, C., 2014).Ces populations ont apporté en Europe deux innovations technologiques majeures que sont la domestication du cheval et la roue et également introduit les langues indo-européennes.

\footnotetext{
${ }^{\mathrm{i}}$ Nous ne mentionnons ici que les théories principales. Il en existe une multitude.

ii les hommes continuent à chasser, à pêcher ou à ramasser des fruits et des plantes sauvages mais il s'agit d'activités d'appoint.

iii « Neolithic package» (Muchaev 1975 Kavkaz na zare bronzovogo veka Nauka. Moskva. (in Russian)

${ }^{\text {iv }}$ Les SNP (Single Nucleotide Polymorphism) sont des mutations d'un seul nucléotide partagées au sein des populations. Les SNPs sont très nombreux $\left(>10^{7}\right.$ par génome humain) et répartis dans tout le génome (environ 1 SNP tous les $300 \mathrm{pb})$. Les SNPs sont référencés dans la base de données dbSNP

(http://www.ncbi.nlm.nih.gov/projects/SNP/). Alors que les SNV (Single Nucleotide Variation) sont des mutations privées et qui de ce fait ne se trouve pas chez tous les individus, ni dans toutes les populations. ${ }^{v}$ C'est-à-dire le passage de materiel génétique d'une lignée dans une autre par le biais d'hybridation et de croisements retours.
}

\section{Références}

Cauvin J. 1994, Naissance des Divinités. Naissance de l'Agriculture. La Révolution des Symboles au Néolithique, Paris, CNRS Éditions.

Chiaroni J., Underhill PA, Cavalli-Sforza LL. 2009, "Y chromosome diversity, human expansion, drift, and cultural evolution”, Proc Natl Acad Sci USA. 106(48):20174-9.

Childe V. G. 1925, The Dawn of European Civilization. Routledge \& Kegan Paul.

Crubézy E., Braga J., Larrouy G. 2008, Anthropologie biologique. Evolution humaine, Paris, Masson.

Demoule J.-P. 2010, La Révolution néolithique dans le monde, Paris, CNRS Éditions.

Fu Q., Li H., Moorjani P., Jay F., Slepchenko S.M., Bondarev A.A., Johnson P.L., AximuPetri A., Prüfer K., de Filippo C., Meyer M., Zwyns N., Salazar-García D.C., Kuzmin Y.V,. Keates S.G., Kosintsev P.A., Razhev D.I., Richards M.P., Peristov N.V., Lachmann M., Douka K., Higham T.F., Slatkin M., Hublin J.J., Reich D., Kelso J., Viola T.B., Pääbo S. 2014, « Genome sequence of a 45,000-year-old modern human from western Siberia », dans Nature, ${ }^{\circ}$ 514, vol. 7523: 445-9. 
Fu Q., Posth C., Hajdinjak M., Petr M., Mallick S., Fernandes D., Furtwängler A., Haak W., Meyer M., Mittnik A., Nickel B., Peltzer A., Rohland N., Slon V., Talamo S., Lazaridis I., Lipson M., Mathieson I., Schiffels S., Skoglund P., Derevianko A.P., Drozdov N., Slavinsky V., Tsybankov A., Cremonesi R.G., Mallegni F., Gély B., Vacca E., Morales M.R., Straus L.G., Neu-gebauer-Maresch C., Teschler-Nicola M., Constantin S., Moldovan O.T., Benazzi S., Peresani M., Coppola D., Lari M., Ricci S., Ronchitelli A., Valentin F., Thevenet C., Wehrberger K., Grigorescu D., Rougier H., Crevecoeur I., Flas D., Semal P., Mannino M.A., Cupillard C., Bocherens H., Conard N.J., Harvati K., Moiseyev V., Drucker D.G., Svoboda J., Richards M.P., Cara $\neg$ melli D., Pinhasi R., Kelso J., Patterson N., Krause J., Pääbo S., Reich D. 2016, «The genetic history of Ice Age Europe » dans Nature, ${ }^{\circ}$ 534, vol. $7606: 200-205$.

Gallego-Llorente M., Connell S., Jones E.R., Merrett D.C., Jeon Y., Eriksson A., Siska V., Gamba C., Meiklejohn C., Beyer R., Jeon S., Cho Y.S., Hofreiter M., Bhak J., Manica A., Pinhasi R.. 2016, « The genetics of an early Neolithic pastoralist from the Zagros, Iran », dans Sci Rep. ${ }^{\circ} 6: 31326$.

Gamba C., Jones E.R., Teasdale M.D.., McLauᄀghlin R.L.., Gonzalez-Fortes G.., Mattiangeli V., Domboróczki L., Kővári I., Pap I., Anders A., Whittle A., Dani J., Raczky P., Higham T.F., Hofreiter M., Bradley D.G., Pinhasi R. 2014, « Genome flux and stasis in a five millennium transect of European prehistory » dans Nature Communica $\neg$ tions $n^{\circ}$ 5: 5257.

Guilaine J. 1996, La Mer Partagée : La Méditerranée avant l'écriture 7000-2000 avant JésusChrist, Paris, Hachette.

Jeunesse C. 2018, «Big men, chefferies ou démocratie primitive ? Quels types de sociétés dans le Néolithique de la France », dans Guilaine J., Garcia D. (éds.), La Protohistoire de la France, Paris, Hermann, pp. 171-185.

Haak W., Lazaridis I., Patterson N., Rohland N., Mallick S., Llamas B., Brandt G., Nordenfelt S., Harney E., Stewardson K., Fu Q., Mittnik A., Bánffy E., Economou C., Francken M., Friederich S., Pena R.G., Hallgren F., Khartanovich V., Khokhlov A., Kunst M., Kuznetsov P., Meller H., Mochalov O., Moiseyev V., Nicklisch N., Pichler S.L., Risch R., Rojo Guerra M.A., Roth C., Szécsényi-Nagy A., Wahl J., Meyer M., Krause J., Brown D., Anthony D., Cooper A., Alt K.W., Reich D. 2015, « Massive migration from the steppe was a source for Indo-European languages in Europe » dans Nature, $n^{\circ}$ 522, vol. $7555: 207-211$.

Hofmanová Z., Kreutzer S., Hellenthal G., Sell C., Diekmann Y., Díez-Del-Molino D., van Dorp L., López S., Kousathanas A., Link V., Kirsanow K., Cassidy L.M., Martiniano R., Strobel M., Scheu A., Kotsakis K., Halstead P., Triantaphyllou S., Kyparissi-Apostolika N., Urem-Kotsou D., Ziota C., Adaktylou F., Gopalan S., Bobo DM., Winkelbach L., Blöcher J., Unterländer M., Leuenberger C., Çilingiroğlu Ç., Horejs B., Gerritsen F., Shennan S.J., Bradley D.G., Currat M., Veeramah K.R., Wegmann D., Thomas M.G., Papageorgopoulou C., Burger J. 2016, « Early farmers from across Europe directly descended from Neolithic Aegeans » dans Proc Natl Acad Sci U S A, n 113, vol. 25: 6886-6891.

Keller A., Graefen A., Ball M., Matzas M., Boisguerin V., Maixner F., Leidinger P., Backes C., Khairat R., Forster M., Stade B., Franke A., Mayer J., Spangler J., McLaunghlin S., Shah M., Lee C., Harkins T.T., Sartori A., Moreno-Estrada A., Henn B., Sikora M., Semino O., Chiaroni J., Rootsi S., Myres N.M., Cabrera V.M., Underhill P.A.,Bustamante C.D., Vigl 
E.E., Samadelli M., Cipollini G., Haas J., Katus H., Constantin S., Moldovan O.T., Benazzi S., Peresani M., Coppola D., Lari M., Ricci S., Ronchitelli A., Valentin F., Thevenet C., Wehrberger K., Grigorescu D., Rougier H., Crevecoeur I., Flas D., Semal P., Mannino M.A., Cupillard C., Bocherens H., Conard N.J., Harvati K., Moiseyev V., Drucker D.G., Svoboda J., Richards M.P., Caraᄀmelli D., Pinhasi R., Kelso J., Patterson N., Krause J., Pääbo S., Reich D. 2016, «The genetic history of Ice Age Europe » dans Nature, ${ }^{\circ}$ 534, vol. $7606: 200-205$.

Gallego-Llorente M., Connell S., Jones E.R., Merrett D.C., Jeon Y., Eriksson A., Siska V., Gamba C., Meiklejohn C., Beyer R., Jeon S., Cho Y.S., Hofreiter M., Bhak J., Manica A., Pinhasi R.. 2016, « The genetics of an early Neolithic pastoralist from the Zagros, Iran », dans Sci Rep. $n^{\circ} 6: 31326$.

Gamba C., Jones E.R., Teasdale M.D.., McLaughlin R.L.., Gonzalez-Fortes G.., Mattianᄀgeli V., Domboróczki L., Kővári I., Pap I., Anders A., Whittle A., Dani J., Raczky P., Higham T.F., Hofreiter M., Bradley D.G., Pinhasi R. 2014, « Genome flux and stasis in a five millennium transect of European prehistory » dans Nature Communica $\neg$ tions $n^{\circ}$ 5: 5257.

Guilaine J. 1996, La Mer Partagée : La Méditerranée avant l'écriture 7000-2000 avant JésusChrist, Paris, Hachette.

Jeunesse C. 2018, «Big men, chefferies ou démocratie primitive ? Quels types de sociétés dans le Néolithique de la France », dans Guilaine J., Garcia D. (éds.), La Protohistoire de la France, Paris, Hermann, pp. 171-185.

Haak W., Lazaridis I., Patterson N., Rohland N., Mallick S., Llamas B., Brandt G., Nordenfelt S., Harney E., Stewardson K., Fu Q., Mittnik A., Bánffy E., Economou C., Francken M., Friederich S., Pena R.G., Hallgren F., Khartanovich V., Khokhlov A., Kunst M., Kuznetsov P., Meller H., Mochalov O., Moiseyev V., Nicklisch N., Pichler S.L., Risch R., Rojo Guerra M.A., Roth C., Szécsényi-Nagy A., Wahl J., Meyer M., Krause J., Brown D., Anthony D., Cooper A., Alt K.W., Reich D. 2015, « Massive migration from the steppe was a source for Indo-European languages in Europe » dans Nature, $n^{\circ}$ 522, vol. $7555: 207-211$.

Hofmanová Z., Kreutzer S., Hellenthal G., Sell C., Diekmann Y., Díez-Del-Molino D., van Dorp L., López S., Kousathanas A., Link V., Kirsanow K., Cassidy L.M., Marרtiniano R., Strobel M., Scheu A., Kotsakis K., Halstead P., Triantaphyllou S., Kypa $r$ rissi-Apostolika N., Urem-Kotsou D., Ziota C., Adaktylou F., Gopalan S., Bobo DM., Winkelbach L., Blöcher J., Unterländer M., Leuenberger C., Çilingiroğlu Ç., Horejs B., Gerritsen F., Shennan S.J., Bradley D.G., Currat M., Veeramah K.R., Wegmann D., Thomas M.G., Papageorgopoulou C., Burger J. 2016, « Early farmers from across Europe directly descended from Neolithic Aegeans »dans Proc Natl Acad Sci U S A, n 113, vol. 25: 6886-6891.

Keller A., Graefen A., Ball M., Matzas M., Boisguerin V., Maixner F., Leidinger P., Backes C., Khairat R., Forster M., Stade B., Franke A., Mayer J., Spangler J., McLau $g$ ghlin S., Shah M., Lee C., Harkins T.T., Sartori A., Moreno-Estrada A., Henn B., Sikora M., Semino O., Chiaroni J., Rootsi S., Myres N.M., Cabrera V.M., Underhill P.A.,Bustamante C.D., Vigl E.E., Sama $\neg$ delli M., Cipollini G., Haas J., Katus H., O’Connor B.D., Carlson M.R., Meder B., Blin N., Meese E., Pusch C.M., Zink A. 2012, « New insights into the Tyrolean Ice $\neg$ man's origin and phenotype as inferred by whole-genome sequencing » dans Nature

Communications, $\mathrm{n}^{\circ}$ 3: 698 . 
Kılınç GM., Koptekin D., Atakuman Ç., Sümer A.P., Dönertaş H.M., Yaka R., Bilgin C.C., Büyükkarakaya A.M., Baird D., Altınışık E., Flegontov P., Götherström A., Togan İ., Somel M. 2017, «Archaeogenomic analysis of the first steps of Neolithization in Anatolia and the Aegean »dans Proc Biol Sci. $n^{\circ} 284: 1867$.

King R.J. \& Underhill P.A. 2018, "Modern DNA and the Ancient Mediterranean" dans Walter Scheidel (éd.), The Science of Roman History: biology, climate, and the future of the past, Princeton University Press.

Lacan M., Keyser C., Ricaut F.X., Brucato N., Duranthon F., Guilaine J., Crubézy E., Ludes B. 2011, «Ancient DNA reveals male diffusion through the Neolithic Mediterranean route » dans Proc Natl Acad Sci U S A, n 108, vol. 24: 9788-9791.

Larsen C.S. 2010, « The agricultural revo $\neg$ lution as environmental catastrophe : Implications for health and lifestyle in the Holocene », dans Quaternary International $n^{\circ} 150$, vol. 1 : 12 20 .

Mathieson I., Lazaridis I., Rohland N., Malᄀlick S., Patterson N., Roodenberg S.A., Harney E., Stewardson K., Fernandes D., Novak M., Sirak K., Gamba C., Jones E.R., Llamas B., Dryomov S., Pickrell J., Arsuaga J.L., de Castro J.M., Carbonell E., Gerritsen F., Khokhlov A., Kuznetsov P., Lozano M., Meller H., Mochalov O., Moiseyev V., Guerra M.A., Roodenberg J., Vergès J.M., Krause J., Cooper A., Alt K.W., Brown D., Anthony D., Lalueza-Fox C., Haak W., Pinhasi R., Reich D. 2015, « Genome-wide patterns of selection in 230 ancient Eurasians » dans Nature, $n^{\circ}$ 528, vol. $7583: 499-503$.

Mendez F.L., Krahn T., Schrack B., Krahn A.M., Veeramah K.R., Woerner A.E., Fomine F.L., Bradman N., Thomas M.G., Karafet T.M., Hammer M.F. 2013, « An African American paternal lineage adds an extremely ancient root to the human $\mathrm{Y}$ chromosome phylogenetic tree » dans Am. J. Hum. Genet., $n^{\circ} 92:$ 454-459.

Mithen S. 2006, After the ice: A global human history, 20,000-5,000 BC, Harvard University Press.

Olalde I., Allentoft M.E., Sánchez-Quinto F., Santpere G., Chiang C.W., DeGiorgio M., Prado-Martinez J., Rodríguez J.A., Rasmussen S., Quilez J., Ramírez O., Marigorta U.M., Fernández-Callejo M., Prada M.E., Encinas J.M., Nielsen R., Netea M.G., Novembre J., Sturm R.A., Sabeti P., Marquès-Bonet T., Navarro A., Willerslev E., Lalueza-Fox C. 2014. « Derived immune and ancestral pigmentation alleles in a 7,000-year-old Mesolithic European $\gg$ dans Nature, $\mathrm{n}^{\circ}$ 507, vol. $7491:$ 225-228.

Quintana-Murci L. \& Patin E. 2018, « L'apport de la génétique à l'histoire d'Homo sapiens. Une histoire des civilisations », dans Demoule J.P., Garcia D., Schnapp A. (éds.), Une histoire des civilisations, Paris, La découverte INRAP.

Poznik G. D., Xue Y., Mendez F.L., Willems T.F., Massaia A., Wilson Sayres M.A., Ayub

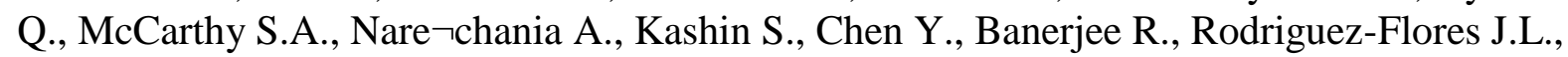
Cerezo M., Shao H., Gymrek M., Malhotra A., Louzada S., Desalle R., Ritchie G.R., Cerveira E., Fitzgerald T.W., Garrison E., Marcketta A., Mittelman D., Romanovitch M., Zhang C., 
Zheng-Bradley X., Abecasis G.R., McCarᄀroll S.A., Flicek P., Underhill P.A., Coin L., Zerbino D.R., Yang F., Lee C., Clarke L., Auton A., Erlich Y., Handsaker RE; 1000 Genomes Project Consortium., Bustamante CD., Tyler-Smith C. 2016, «Punctuated bursts in human male demography inferred from 1,244 worldwide Y chromosome sequences » dans Nature Genetics n 48 , vol. $6: 593-599$.

Seguin-Orlando, A., Korneliussen T.S., Sikora M., Malaspinas A.S., Manica A., Moltke I., Albrechtsen A., Ko A., Marga $\neg$ ryan A., Moiseyev V., Goebel T., Westaway M., Lambert D., Khartanovich V., Wall J.D., Nigst P.R., Foley R.A., Lahr M.M., Nielsen R., Orlando L., Willerslev E. 2014. «Paleogenomics: genomic structure in Europeans dating back at least 36,200 years » dans Science, $n^{\circ} 346$, vol. $6213: 1113-1118$.

Ruff C.B., Holt B., Niskanen M., Sladek V., Berner M., Garofalo E., Garvin H.M., Hora M., Junno J.A., Schuplerova E., Vilkama R., Whittey E. 2015, « Gradual decline in mobility with the adoption of food production in Europe », dans Proc Natl Acad Sci U S A, n 9 : 71477152.

Testart A. 2010, «Des crânes et des vautours ou la Guerre oubliée », dans Paléo n 34 (1) : 33-58.

Testart A. 2012, Avant l'histoire : L'évolution des sociétés, de Lascaux à Carnac, Paris, Éditions Gallimard.

Voskarides K., Mazières S., Hadjipanagi D., Di Cristofaro J., Ignatiou A., Stefanou C., King R.J., Underhill P.A., Chiaroni J., Deltas C. 2016. «Y-chromosome phylogeographic analysis of the Greek-Cypriot population reveals elements consistent with Neolithic and Bronze Age settlements » dans Investigative Genetics, $n^{\circ}$ 7: 1-14. 